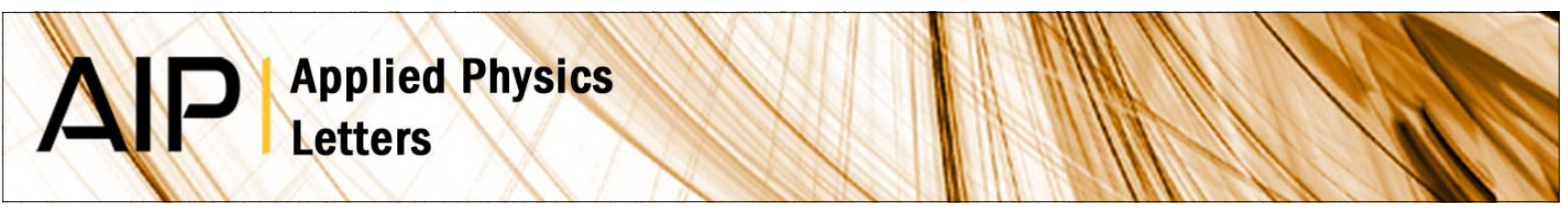

\title{
Semiconducting graphene nanoribbon retains band gap on amorphous or crystalline $\mathrm{SiO} 2$
}

M. Zubaer Hossain

Citation: Appl. Phys. Lett. 99, 183103 (2011); doi: 10.1063/1.3657494

View online: http://dx.doi.org/10.1063/1.3657494

View Table of Contents: http://apl.aip.org/resource/1/APPLAB/v99/i18

Published by the American Institute of Physics.

\section{Related Articles}

Excitation of discrete and continuous spectrum for a surface conductivity model of graphene J. Appl. Phys. 110, 114305 (2011)

Abnormal electronic transport and negative differential resistance of graphene nanoribbons with defects Appl. Phys. Lett. 99, 192102 (2011)

The effect of doping on the energetics and quantum conductance in graphene nanoribbons with a metallocene adsorbate

J. Chem. Phys. 135, 124708 (2011)

The destruction of Landau levels in graphene nanoribbons by magnetic modulation

J. Appl. Phys. 110, 063718 (2011)

Half-metallicity and spin-contamination of the electronic ground state of graphene nanoribbons and related systems: An impossible compromise?

J. Chem. Phys. 135, 104704 (2011)

\section{Additional information on Appl. Phys. Lett.}

Journal Homepage: http://apl.aip.org/

Journal Information: http://apl.aip.org/about/about_the_journal

Top downloads: http://apl.aip.org/features/most_downloaded

Information for Authors: http://apl.aip.org/authors

\section{ADVERTISEMENT}

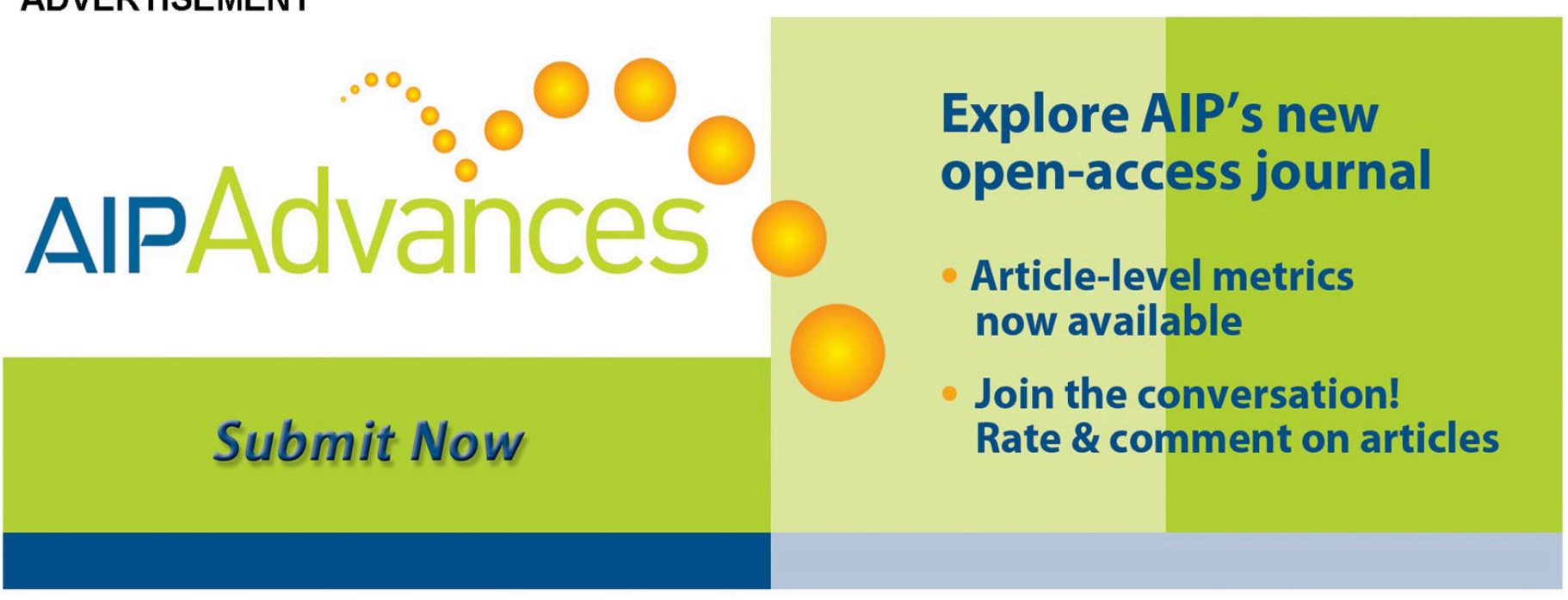




\title{
Semiconducting graphene nanoribbon retains band gap on amorphous or crystalline $\mathrm{SiO}_{2}$
}

\author{
M. Zubaer Hossain ${ }^{\text {a) }}$ \\ Division of Engineering and Applied Science, California Institute of Technology, Pasadena, \\ California 91125, USA
}

(Received 25 August 2011; accepted 10 October 2011; published online 31 October 2011)

\begin{abstract}
Electronic properties of a semiconducting armchair graphene nanoribbon on $\mathrm{SiO}_{2}$ are examined using first-principles calculations and taking into account the van der Waals interaction. Unlike semiconducting carbon nanotubes, which exhibit variations in band gap on $\mathrm{SiO}_{2}$, the nanoribbon is found to retain its band gap on $\mathrm{SiO}_{2}$, regardless of the separation distance or the dielectric's surface type-crystalline or amorphous. The interfacial interaction leads to electron-transfer from the nanoribbon to the dielectric. Moreover, for crystalline $\mathrm{SiO}_{2}$, the quantity of electron-transfer and the binding energy depend strongly on the type of surface termination and weakly on the binding sites. (C) 2011 American Institute of Physics. [doi:10.1063/1.3657494]
\end{abstract}

Graphene nanoribbons (GNRs) show distinct material properties from those of other carbon allotropes. ${ }^{1-4}$ In addition to edge chemistry, their interaction with dielectric surfaces holds particular importance, especially in terms of nanoribbon's potential applications in electronic devices. Owing to one-atom thickness and finite dimension, the susceptibility of a nanoribbon to chemical or electronic degradation arising from interactions with foreign atoms or an interface needs to be understood in detail to harness its full potential.

Graphene- $\mathrm{SiO}_{2},{ }^{5-8} \mathrm{ZGNR}-\mathrm{SiO}_{2},{ }^{9}$ and $\mathrm{CNT}-\mathrm{SiO}_{2},{ }^{10}$ are studied in detail. Here, AGNR-SiO 2 is examined, using density functional theory (DFT) calculations. And it is shown that, unlike semiconducting CNT, AGNR retains its band gap on crystalline or amorphous $\mathrm{SiO}_{2}$. Nonetheless, it has energetic preference on crystalline $\mathrm{SiO}_{2}$ and electrons are transferred to the dielectric, which makes the nanoribbon a $p$-type material on $\mathrm{SiO}_{2}$.

The interfacial interactions are investigated for both crystalline $\mathrm{SiO}_{2}\left(c-\mathrm{SiO}_{2}\right)$ and amorphous $\mathrm{SiO}_{2}\left(a-\mathrm{SiO}_{2}\right)$, where the crystal structure of $\alpha$-quartz is used for the $c-\mathrm{SiO}_{2}$ structure, and $a-\mathrm{SiO}_{2}$ is prepared by annealing and cooling $c-\mathrm{SiO}_{2}$ through molecular dynamics simulations. For $c-\mathrm{SiO}_{2}$, because of symmetry, only a few interfacial configurations $(\mathrm{P}, \mathrm{Q}, \mathrm{R})$ are sufficient to determine the generic behavior of an interface. ${ }^{5}$ As depicted in Fig. S1 in the supplementary material, ${ }^{11}$ in $\mathrm{P}$ configuration, the surface atoms (either Si or $\mathrm{O})$ are right below the center of the hexagons, and in $\mathrm{R}$ configuration, they are located right below the carbon atoms. On the other hand, for $a-\mathrm{SiO}_{2}$, which hardly has any symmetric configuration, the nanoribbon is placed at various randomly chosen locations on the $a-\mathrm{SiO}_{2}$ surface and the electronic properties are extracted in an average sense. Unlike graphene, the bonding characteristics between atoms change sharply at the edges of GNRs. ${ }^{12}$ Here, we consider the stable edge configuration $a_{11}$ (as noted in Ref. 12), which is shown to be nonmetallic and nonmagnetic.

The amorphous structure of $\mathrm{SiO}_{2}\left(a-\mathrm{SiO}_{2}\right)$ is prepared by annealing $c-\mathrm{SiO}_{2}$ using the molecular dynamics code

\footnotetext{
${ }^{\text {a)} E l e c t r o n i c ~ m a i l: ~ z u b a e r @ c a l t e c h . e d u . ~}$
}

LAMMPS. ${ }^{13}$ The Tersoff ${ }^{14}$ interatomic potential, parameterized by Munetoh, ${ }^{15,16}$ is used to model Si-O interactions. The atomistic structure of $c-\mathrm{SiO}_{2}$, after raising its temperature to $5000 \mathrm{~K}$ and holding it for $10 \mathrm{ps}$, is cooled to room temperature at a cooling rates of $1.0 \times 10^{11} \mathrm{~K} / \mathrm{s}$. Different annealing temperatures $(4000,5000$, and $6000 \mathrm{~K})$ and cooling rates $\left(1.0 \times 10^{10}, 1.0 \times 10^{11}\right.$, and $\left.1.0 \times 10^{12} \mathrm{~K} / \mathrm{s}\right)$ are chosen to study their effects on the prepared amorphous structure. The conclusions are found not to be affected by them. The resulting $a-\mathrm{SiO}_{2}$ structure is then relaxed using DFT calculations. A comparison of the electronic charge distribution on relaxed $c-\mathrm{SiO}_{2}$ and $a-\mathrm{SiO}_{2}$ surfaces (as shown in Fig. 1) and at different depths from the surface (as depicted in Fig. S2) ${ }^{11}$ demonstrates that the electronic charge distribution in $a-\mathrm{SiO}_{2}$, at any plane parallel to the graphene nanoribbon, is inhomogeneous. However this distribution causes no substantial change to the semiconducting characteristics of the nanoribbon.

The electronic structure calculations are performed with the SIESTA code ${ }^{17}$ using the local density approximations (LDA) (Ref. 18) and vdW-DFT (Ref. 19) exchangecorrelation energy functionals. Following a Troullier and Martins scheme, ${ }^{20}$ the core electrons are replaced by norm conserving pseudopotentials, and the valence electrons are represented by a double-zeta polarized (DZP) numerical atomic basis. Atomic structures are relaxed using a force tolerance of $0.02 \mathrm{eV} / \AA$. Obtained relaxed lattice parameters are: $a=b=4.918 \AA$ and $c=5.407 \AA$ for $c-\mathrm{SiO}_{2} ; \mathrm{C}-\mathrm{C}$ bond length $=1.42 \AA$ and $\mathrm{C}-\mathrm{H}$ bond length $=0.97 \AA$ for $\mathrm{AGNR}$. The length of the $\mathrm{SiO}_{2}$ (0001) surface along the $y$-direction is taken as $8.52 \AA$, which matches with the nanoribbon's length along the periodic direction, ${ }^{5}$ thus causing no significant strain. The total energy for the interface calculations is converged for a $k$-mesh of $\left(k_{x}, k_{y}, k_{z}\right) \equiv(2 \times 12 \times 1)$ which contains $14 k$-points in the irreducible Brillouin zone.

The energy of interaction is calculated for a number of distinct separation distances, as shown in Fig. 2. For any particular interface configuration, the separation distance $d_{0}$ denotes the distance where the interaction energy is a minimum, $E_{0}$. Their values, presented in Table I, indicate a weak site dependent energetic preference $\left(1.9 \mathrm{meV}^{\circ} \AA^{2}\right.$ for the 


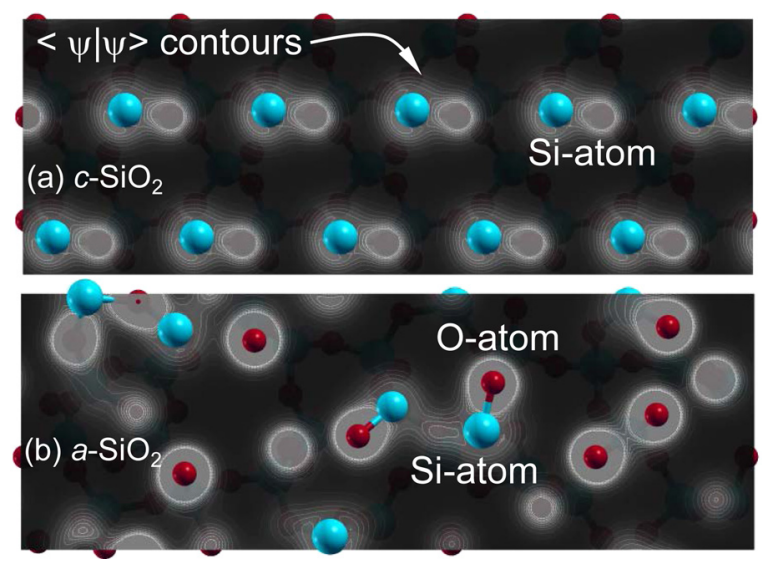

FIG. 1. (Color online) Distribution of electronic charge and atoms on the surface of relaxed (a) $c-\mathrm{SiO}_{2}$ and (b) $a-\mathrm{SiO}_{2}$. The charge is shown for a range of 0.0-0.1 electrons/bohr ${ }^{3}$. The distributions for crystalline $\mathrm{SiO}_{2}$, as shown in (a), remain homogeneous, while for amorphous $\mathrm{SiO}_{2}$, as shown in (b), they become highly inhomogeneous.

O-terminated surface and $5.4 \mathrm{meV} / \AA^{2}$ for the Si-terminated surface) for LDA calculations. On the other hand, vdW calculations show a very small energetic preference. On an average, for $c-\mathrm{SiO}_{2}$, LDA overestimates binding energy, which is the case in general, ${ }^{21}$ by approximately $8.30 \mathrm{meV} / \AA^{2}$ and $11.85 \mathrm{meV} / \AA^{2}$ on $\mathrm{Si}$ - and O-terminated surfaces, respectively. Conversely, it underestimates the equilibrium distance by approximately $0.625 \AA$ and $0.375 \AA$ on $\mathrm{Si}$ - and Oterminated surfaces, respectively. Likewise, on amorphous $\mathrm{SiO}_{2}$, depending on the number of silicon and oxygen atoms interacting with the AGNR, the interaction energy lies within that obtained for the O- and Si-terminated cases for crystalline $\mathrm{SiO}_{2}$.

Analogous to graphene ${ }^{5}$ or $\mathrm{CNT},{ }^{10}$ the interface formation leads to electron-transfer from the graphene nanoribbon to the dielectric, as shown in Fig. 3. The charge-transfer characteristics are investigated by using the Mulliken population analysis, ${ }^{5}$ as implemented in the SIESTA code.

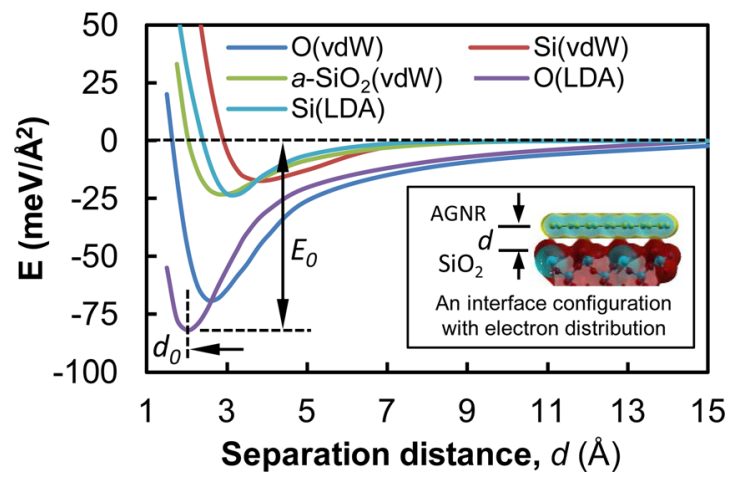

FIG. 2. (Color online) Interaction energy $E$ as a function of separation distance $d$. The $E-d$ behavior for the amorphous dielectric shows that the surface is Si-rich. Nevertheless, all amorphous configurations considered in this work followed an $E$ - $d$ relation within the $E$ - $d$ relations obtained for the $\mathrm{Si}$ and $\mathrm{O}$-terminated $c-\mathrm{SiO}_{2}$ surfaces. Irrespective of the exchange-correlation potential used in the calculations, the interaction energy depends on the type of surface termination. Moreover, for $a-\mathrm{SiO}_{2}$, the interaction energy mainly depends on the relative amount of $\mathrm{O}$ and $\mathrm{Si}$ atoms present on the dielectric surface. The equilibrium distance $\left(d_{0}\right)$ and binding energy $\left(E_{0}\right)$ are marked for AGNR interacting with O-terminated $c-\mathrm{SiO}_{2}$.
TABLE I. Site dependent binding energy, $E_{0}\left(\mathrm{meV} / \AA^{2}\right)$ and equilibrium binding distance, $d_{0}(\AA)$ for the Si- and O-terminated surfaces. The energy is normalized by the area of the nanoribbon.

\begin{tabular}{lccccc}
\hline \hline & $\mathrm{O}(\mathrm{P})$ & $\mathrm{O}(\mathrm{R})$ & $\mathrm{Si}(\mathrm{P})$ & $\mathrm{Si}(\mathrm{R})$ & $a-\mathrm{SiO}_{2}$ \\
\hline$E_{0}(\mathrm{LDA})$ & 80.0 & 81.9 & 28.8 & 23.4 & - \\
$E_{0}(\mathrm{vdW})$ & 69.0 & 69.2 & 18.6 & 17.2 & 24.0 \\
$d_{0}(\mathrm{LDA})$ & 2.25 & 2.0 & 3.0 & 3.25 & - \\
$d_{0}(\mathrm{vdW})$ & 2.50 & 2.5 & 3.5 & 4.00 & 3.0 \\
\hline \hline
\end{tabular}

The relation between the quantities of charge transfer across the interface and separation distance is well fitted by a simple mathematical form ${ }^{5}$

$$
\Delta Q=\alpha d^{-\beta}
$$

where $\Delta Q$ (electrons $/ \AA$ or $\mathrm{e} / \AA$ ) is the amount of charge transferred across the interface and $\alpha, \beta$ are the fitting parameters. The values of the fitting parameters shown in Table II are determined by using the curve fitting utility cftool in MATLAB. For the O-terminated surface the quantity of charge-transfer is a maximum in the $\mathrm{R}$ configuration, whereas for the $\mathrm{Si}$ terminated surface, the charge-transfer characteristics in $\mathrm{P}$ and $\mathrm{R}$ configurations become very similar.

Irrespective of the surface type, electrons are transferred from the nanoribbon to the dielectric making the nanoribbon a $p$-type material on $\mathrm{SiO}_{2}$. The total number of transferred electrons depends on the number of $\mathrm{C}-\mathrm{Si}$ or $\mathrm{C}-\mathrm{O}$ channels formed at the interface. The atomic spacing ( $\mathrm{Si}-\mathrm{Si}$ for the Si-terminated surface, O-O for the O-terminated surface, or $\mathrm{Si}-\mathrm{O}$ for $a-\mathrm{SiO}_{2}$ ) between the surface atoms is larger than the nanoribbon's $\mathrm{C}-\mathrm{C}$ bond length. It can be argued that the interactions between the channels or binding sites are negligible and the channels can effectively act independently to transfer electrons to the dielectric. The total charge-transfer can, thus, be determined by summing contributions from all the channels that are formed across the interface.

In spite of the above mentioned energetics or chargetransfer characteristics as well as the differences noted in the

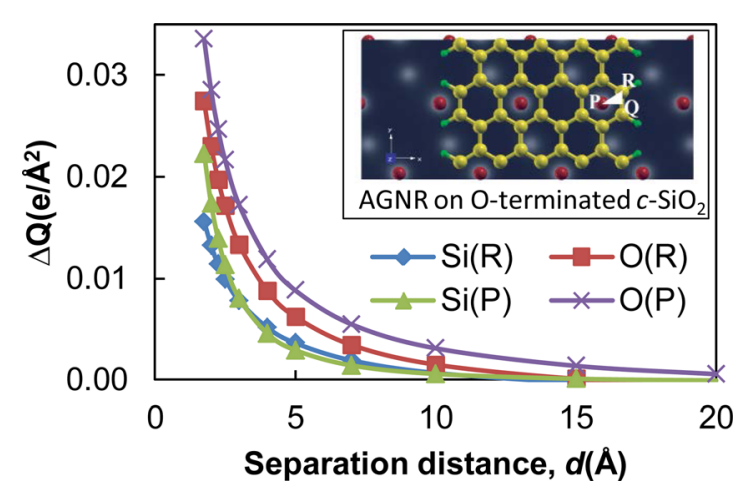

FIG. 3. (Color online) Charge-transfer $\Delta Q$ as a function of separation distance, $d$ for the $\mathrm{Si}(\mathrm{R}), \mathrm{O}(\mathrm{R}), \mathrm{Si}(\mathrm{P})$, and $\mathrm{O}(\mathrm{P})$, where $\mathrm{Si}$ or O refers to termination type, and $\mathrm{P}$ or $\mathrm{R}$ refers to site type. The atomic configuration in the inset shows the high symmetry positions, $\mathrm{P}, \mathrm{Q}, \mathrm{R}$ for O-terminated $c-\mathrm{SiO}_{2}$ surface. The charge-transfer from AGNR to the O-terminated surface is higher than that to the Si-terminated surface. Binding site has negligible effect on charge-transfer for the Si-terminated surface. However, for the O-terminated surface, the effect is substantial. 
TABLE II. Parameters describing the power law relation between chargetransfer and separation distance on sites $\mathrm{P}$ and $\mathrm{R}$ in $\mathrm{Si}$ - and O-terminated dielectric surfaces. The unit of the parameter $\alpha$ is electrons $/ \AA^{2}$.

\begin{tabular}{ccccc}
\hline \hline & $\mathrm{O}(\mathrm{P})$ & $\mathrm{O}(\mathrm{R})$ & $\mathrm{Si}(\mathrm{P})$ & $\mathrm{Si}(\mathrm{R})$ \\
\hline$\alpha$ & $7.0 \times 10^{-3}$ & $6.3 \times 10^{-3}$ & $6.53 \times 10^{-3}$ & $3.58 \times 10^{-3}$ \\
$B$ & 1.296 & 1.448 & 1.916 & 1.434 \\
\hline \hline
\end{tabular}

electronic charge distributions on amorphous $\mathrm{SiO}_{2}$, the electronic characteristics around the Fermi energy is found to be unaffected in all circumstances. The perturbation caused by interface formation to the total density of states of an isolated AGNR is the sum of the partial density of states (PDOS) for all the $\mathrm{C}$ atoms in the AGNR. The total DOS of the AGNR, for a several surface configurations considered here, are shown in Fig. 4.

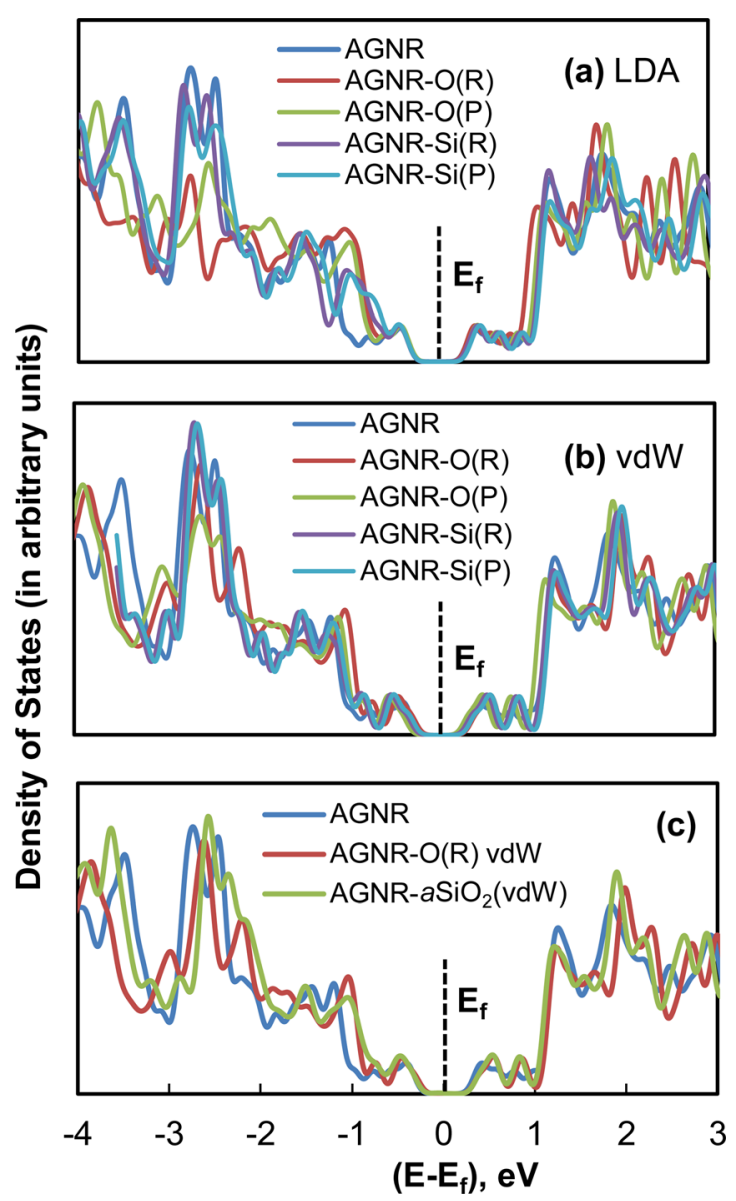

FIG. 4. (Color online) Effect of interface formation on AGNR's DOS for (a) LDA-DFT calculations and (b) vdW-DFT calculations. (c) compares the electronic states of pristine AGNR, AGNR with $a-\mathrm{SiO}_{2}$, and AGNR with $c-\mathrm{SiO}_{2}$ interface. It is evident that the band gap is all cases is unaffected by the interaction at the interface. The Fermi energy, $E_{\mathrm{f}}$, is marked by the dotted lines.
For the O-terminated surface, where the interaction with the nanoribbon is stronger, AGNRs DOS (away from the Fermi energy) get affected the most. However, the changes manifested in the DOS plots for the O-terminated surface goes away as the separation distance is increased. Likewise, for the Si-terminated surface, the electronic states of the nanoribbon remain mainly unaltered. Moreover, it is remarkable that even in $a-\mathrm{SiO}_{2}$ (where a significant charge inhomogeneity is present), no defect states are created within the band gap. Despite the minor changes to the electronic states of the nanoribbon, the band gap is unchanged in all cases: (a) $\mathrm{O}$ - or Si-terminated $c-\mathrm{SiO}_{2}$, (b) nanoribbon on various binding sites on $c-\mathrm{SiO}_{2}$, (c) $a-\mathrm{SiO}_{2}$ or $c-\mathrm{SiO}_{2}$, and (d) LDA-DFT and vdW-DFT.

In summary, the energetics as well as the electrontransfer characteristics of AGNR are found to be influenced by interface formation. Even though the electronic states of the nanoribbon are affected (especially away from the Fermi energy) by interface formation, the band gap of the nanoribbon is unaltered for $c-\mathrm{SiO}_{2}$ or $a-\mathrm{SiO}_{2}$.

This work used the Extreme Science and Engineering Discovery Environment (XSEDE), which is supported by National Science Foundation grant number OCI-1053575.

${ }^{1}$ A. Chuvilin, E. Bichoutskaia, M. C. Gimenez-Lopez, T. W. Chamberlain, G. A. Rance, N. Kuganathan, J. Biskupek, U. Kaiser, and A. N. Khlobystov, Nature Mater. 10, 687 (2011).

${ }^{2}$ X. L. Li, X. Wang, L. Zhang, S. Lee, and H. Dai, Science 319, 1229 (2008).

${ }^{3}$ X. Wang, Y. Ouyang, X. Li, H. Wang, J. Guo, and H. Dai, Phys. Rev. Lett. 100, 206803 (2008).

${ }^{4}$ M. Y. Han, B. Ozyilmaz, Y. B. Zhang, and P. Kim, Phys. Rev. Lett. 98, 206805 (2007).

${ }^{5}$ M. Z. Hossain, Appl. Phys. Lett. 95, 143125 (2009).

${ }^{6}$ N. T. Cuong, M. Otani, and S. Okada, Phys. Rev. Lett. 106, 106801 (2011).

${ }^{7}$ A. N. Rudenko, F. J. Keil, M. I. Katsnelson, and A. I. Lichtenstein, Phys. Rev. B 84, 085438 (2011)

${ }^{8}$ H. E. Romero, N. Shen, P. Joshi, H. R. Guterrez, S. A. Tadigadapa, J. O. Sofo, and P. C. Eklund, ACS Nano 2, 2037 (2008).

${ }^{9}$ D. M. Zhang, Z. Li, J. F. Miao, and J. J. Jiang, Nanotechnology 22, 265702 (2011).

${ }^{10}$ M. Z. Hossain, Appl. Phys. Lett. 96, 053118 (2010).

${ }^{11}$ See supplementary material at http://dx.doi.org/10.1063/1.3657494 for detailed atomic structure and electronic charge distribution.

${ }^{12}$ T. Wassman, A. P. Seitsonen, A. M. Saitta, M. Lazzeri, and F. Mauri, Phys. Rev. Lett. 101, 096402 (2008).

${ }^{13}$ S. Plimpton, J. Comput. Phys. 117, 1 (1995).

${ }^{14}$ J. Tersoff, Phys. Rev. B 39, 5566 (1989).

${ }^{15}$ S. Munetoh, T. Motooka, K. Moriguchi, and A. Shintani, Comput. Mater. Sci. 39, 334 (2007).

${ }^{16}$ T. Mchedidze, T. Arguirov, S. Kouteva-Arguirova, and M Kittler, J. Appl. Phys. 107, 124302 (2010); E. Lampin and C. Krzeminski, ibid. 109, 123509 (2011).

${ }^{17}$ J. M. Soler, E. Artacho, J. D. Gale, A. Garcia, J. Junquera, P. Ordejon, and D. Sanchez-Portal, J. Phys. Condens. Matter 14, 2745 (2002).

${ }^{18}$ J. P. Perdew and A. Zunger, Phys. Rev. B 23, 5048 (1981).

${ }^{19}$ M. Dion, H. Rydberg, E. Schroder, D. C. Langreth, and B. I. Lundqvist, Phys. Rev. Lett. 92, 246401 (2004).

${ }^{20}$ N. Troullier and J. L. Martins, Phys. Rev. B 43, 1993 (1991).

${ }^{21}$ A. Tkatchenko, L. Romaner, O. T. Hofmann, E. Zojer, C. A. Draxl, and M. Scheffler, MRS Bull. 35, 435 (2010). 